\title{
The Association of Flow-Mediated Dilatation and Blood Parameters in Primary Raynaud's Phenomenon
}

\author{
Süheyla Uzun $\mathbb{D}^{1}$ and İlker Kaya $\mathbb{D}^{2}$ \\ ${ }^{1}$ Department of Internal Medicine, Gaziosmanpasa University, Tokat, Turkey \\ ${ }^{2}$ Department of Cardiovascular Surgery, Tokat State Hospital, Tokat, Turkey \\ Correspondence should be addressed to İlker Kaya; kayalker60@gmail.com
}

Received 7 October 2021; Accepted 30 November 2021; Published 31 January 2022

Academic Editor: Khaled Saad

Copyright (c) 2022 Süheyla Uzun and İlker Kaya. This is an open access article distributed under the Creative Commons Attribution License, which permits unrestricted use, distribution, and reproduction in any medium, provided the original work is properly cited.

\begin{abstract}
Introduction. Raynaud's phenomenon (RP) is a multifactorial disorder. If any underlying disease cannot be determined to be responsible for RP, then it is considered to be the primary RP (pRP). We aimed to investigate the differences between laboratory markers and impaired endothelial function in pRP. Materials and Methods. Forty-two pRP patients and 30 healthy individuals were included as the study and control groups, respectively. The endothelial function was evaluated with flow-mediated dilatation (FMD) of the brachial artery. The blood samples were obtained from both groups, and white blood cell (WBC), hemoglobin, platelets, mean platelet volume (MPV), creatinine, alanine aminotransferase (ALT), aspartate aminotransferase (AST), D-dimer, fibrinogen, albumin, fibrinogen-to-albumin ratio (FAR), neutrophil-to-lymphocyte ratio (NLR), D-dimer-to-albumin ratio (DDAR), and monocyte chemoattractant protein-1 (MCP-1) parameters were studied. The blood parameters and FMD values obtained were compared between groups. Results. The groups were similar in regard to age, gender, and smoking history $(p<0.05)$. There was no difference between the two groups in regard to hemoglobin, platelet, MPV, creatinine, ALT, D-dimer, albumin, FAR, NLR, and DDAR levels $(p<0.05)$. AST levels were slightly higher in the pRP group $(p=0.027)$. Markedly increased WBC, fibrinogen, MPV, and MCP-1 values were detected in the pRP group $(p=0.001)$, as well as higher abnormal FMD responses $(p=0.001)$. There was a direct correlation between abnormal FMD response and serum MCP-1 values in patients with pRP $\left(R: 0.308, R^{2}: 0.095, p: 0.044\right)$. Conclusion. It seems to be that MCP-1 levels are higher in patients with pRP, and increased values of MCP-1 levels seem to be related to impaired endothelial functions.
\end{abstract}

\section{Introduction}

Raynaud's phenomenon (RP) is a reversible vasospastic disorder that may be provoked by exposure to cold or by emotional stress. It involves extremity arteries and presents with ischemic symptoms in the extremities [1]. RP can be a preliminary finding of some diseases, in which case it is referred to as secondary RP (sRP). The disorder can be related to traumas or microtraumas resulting from prolonged usage of vibratory tools, exposure to some chemicals, and autoimmune rheumatic or connective tissue diseases, such as systemic sclerosis. If it progresses, it can result in digital ulcers or microamputations [1-3]. However, RP can also occur in the absence of underlying disease or unknown etiology, in which case it is referred to as primary RP (pRP). This sort of pRP is commonly linked to atmospheric conditions, and therefore, the symptoms are dramatically relieved when exposure to the cause is terminated $[4,5]$. In the primary type, there is no autoimmunity-associated marker or reaction and therefore, a comprehensive anamnesis should be taken and a physical and laboratory examination should be performed in every case of RP, since after excluding suspected pathologies, the RP cases are often classified as primary type $[1,5]$.

The essential finding of $\mathrm{pRP}$ is aggravation of symptoms through exposure to cold and the detection of color changes in the skin, which turns white, blue, or both. The diagnosis is clarified after exclusion of possible underlying diseases, such 
as checking nails with capillaroscopy or a laboratory panels such as complete blood counts, inflammatory parameters, metabolic panel, antibodies, and enzymes. Avoiding contact with cold and stress is suggested for these patients, and lifestyle changes such as reducing smoking and caffeine use are important. In addition, some medications can be applied [1-5]. Some patients have borderline symptoms without physical findings, similar to a feeling of cold within the extremities. In this patient group, management is more challenging when no finding apart from anamnesis has been determined and there is an absence of parameters for possible detection [6, 7]. In particular, an abnormal capillaroscopic finding for differentiation of RP is discriminative for secondary RP. Thus, nailfold capillaroscopies are losing their role as potential predictors of pRP.

In recent years, changes in blood parameters have been frequently examined to better define or differentiate borderline or suspected diseases. In particular, inflammatory biomarkers have become quite popular. Investigation of mean platelet volume (MPV) and neutrophil-to-lymphocyte ratio (NLR) levels is a good example of these current studies. NLR has also become an inflammatory predictor that is currently used in clinics. It has been predicted that NLR may be a valuable marker in many systemic inflammatory processes [8]. Moreover, it has been emphasized that these parameters may be predictive in vascular diseases. While MPV values are predicted to have a diagnostic predictive value in patients with thromboangiitis obliterans involving the distal vascular bed, which is a disease very similar to RP, on the other hand, NLR values were also found to be associated with intima-media thickness in patients with Behcet's disease $[9,10]$. In addition, monocyte chemoattractant protein-1 (MCP-1) is a serum inflammation parameter that has been investigated in similar conditions and it has been less studied in vascular system diseases compared to other parameters [11].

The aim of this study was to investigate the possible predictors for $\mathrm{pRP}$. In the scope of this objective, flowmediated dilatation (FMD), routine blood markers, and MCP-1 levels were compared between pRP patients and healthy individuals.

1.1. What Is Already Known about This Topic? Raynaud's phenomenon (RP) is a reversible vasospastic disorder that may be provoked by cold or emotional stress. This phenomenon can occur without underlying disease or unknown etiology, in which case it is referred to as primary RP $(\mathrm{pRP})$. The essential finding of $\mathrm{pRP}$ is an aggravation of symptoms by exposure to cold and the detection of skin color changes in the extremities (turns white, blue, or both). The diagnosis is clarified after exclusion of possible underlying diseases by checking nail capillaroscopy and laboratory panels, such as complete blood counts, inflammatory parameters, metabolic panel, antibodies, and enzymes. However, any specific laboratory marker has not been determined for diagnosing pRP. Therefore, researchers have tried to establish an objective scientific laboratory marker that is highly specific to pRP.
1.2. What This Study Proposes? To the best of our knowledge, this is the first study examining the relationship between FMD response (impaired endothelial response) and blood parameters, in patients with pRP. This is also the first report regarding the relationship between impaired endothelial response and MCP-1 levels in pRP. Our results have indicated that patients with pRP have a higher impaired endothelial response to ischemia and the incremental levels of serum WBC, MPV, MCP-1, and fibrinogen values were detected in the pRP group. Moreover, a moderate direct correlation was found between abnormal FMD response and serum MCP-1 values.

\section{Materials and Methods}

The study steps were designed according to the Declaration of Helsinki, and ethical approval was obtained from the Local Ethics Committee of the University. Signed informed consent was obtained from all participants. This study was designed as a prospective, case-control study.

Patients over 50 years of age (because of increased risk of atherosclerotic occlusive arterial diseases), patients with a past medical history with trauma, thoracic outlet syndrome, secondary RP (malignity, vasculitis, systemic inflammatory disease, autoimmune diseases, etc.), and anti-inflammatory or corticosteroid usage, and patients with accompanying diabetes, hypertension, and familial hyperlipidemia were all excluded from the study. After exclusion from secondary RP, a total of 43 patients were included, who admitted to the cardiovascular surgery clinic with RP symptoms (bruising and cold hands or feet, provoked by cold) and were diagnosed as primary RP (Figure 1). The control group was formed from healthy individuals who admitted to the hospital for a routine checkup, without cardiovascular symptoms.

2.1. Evaluation of Flow-Mediated Dilatation (FMD). The FMD evaluation was performed on each participant, in the early morning after an overnight fasting period in a standard ultrasound room by an expert sonographer, before starting other steps of the study (blood sampling), the whole as prescribed in the literature $[12,13]$. An Android-compatible linear transducer (Philips Lumify L12-4 (12-4 mHz); Philips Ultrasound Inc., Bothell, WA) was used to perform evaluation of vessel diameters. The normal diameter of the brachial artery was measured over approximately $5 \mathrm{~cm}$ of the antecubital fossa, and thereupon, the evaluation point was marked. The sphygmomanometer cuff was placed to the proximal site of the evaluation point and inflated to above $50 \mathrm{mmHg}$ of normal blood pressure for five minutes, as prescribed in the literature $[12,13]$. After 5 minutes of ischemia period, the cuff was deflated and the ultrasound probe was placed on the marked point, for measurement of postischemic diameter of the brachial artery. The enlargement of diameter equal or above $5 \%$ was accepted as a normal response, whereas diameter lower than 5\% was accepted as an abnormal vascular response to ischemia, indicating an abnormal endothelial function $[12,13]$. 
2.2. Blood Sampling. After evaluation of the FMD response, the routine $10 \mathrm{ml}$ venous blood samples were obtained from each participant. Following centrifugation, routine laboratory parameters (white blood cell (WBC) $\left(10^{-3} / \mathrm{uL}\right.$ ), hemoglobin $(\mathrm{g} / \mathrm{dL})$, platelet $\left(10^{-3} / \mathrm{uL}\right)$, mean platelet volume (MPV) (fL), creatinine $(\mathrm{mg} / \mathrm{dL})$, alanine aminotransferase (ALT) (IU/L), aspartate aminotransferase (AST) (IU/L), D-dimer $(\mathrm{ng} / \mathrm{L})$, fibrinogen $(\mu \mathrm{g} / \mathrm{ml})$, albumin $(\mathrm{g} / \mathrm{dL})$, fibrinogen-to-albumin ratio (FAR) (\%), neutrophil-to-lymphocyte ratio (NLR) (\%), D-dimer-to-albumin ratio (DDAR) (\%), and monocyte chemoattractant protein-1 (MCP-1) $(\mathrm{pg} / \mathrm{ml})$ levels were studied from the acquired serum samples. The commercially available human MCP-1 ELISA kit (SUNLONG, Sun Long Biotech Co., LTD) was used for determining MCP-1 levels (SUNLONG) as described in previous reports [11].

2.3. Statistical Analysis. In this study, sample collection calculation was made with power analysis by using GPower 3.1.9.4 program to calculate sample size and $\alpha=0.05$, $\beta=0.10$, and effect size $=0.776$ were chosen. Since the MCP1 parameter was thought to be the most decisive data in the study, these values from the reference article were used to calculate the effect size [14]. The nominal data analysis was made using the Yates-corrected chi-square test. A comparison of parametric data between the two groups was made using Student's $t$-test. The Pearson correlation coefficients were used to compare the relation of parameters with FMD. The SPSS 17.0 (SPSS Inc., Chicago, IL, USA) statistical software program was used for all statistical analysis, and a $p$ value less than 0.05 was considered significant. By utilizing receiver operating characteristic (ROC) analysis, the area under the curve (AUC) with lower and upper bounds was used to calculate the cutoff value, specificity, and sensitivity. In order to make a Raynaud phenomenon predictive analysis with abnormal FMD, WBC, and MCP-1 values, the cutoff values obtained in the ROC analysis and the variables were categorized and a multiple binary regression analysis was performed. Nagelkerke's $R^{2}$ determination coefficient was used to evaluate the predictive ability of the model [15].

\section{Results}

The groups were similar $(p>0.05)$ in regard to demographical variables (age, gender, and smoking history), but markedly higher abnormal FMD response rates were detected in the primary RP patient group $(p=0.001)$. The comparison of demographical, clinical (FMD), and laboratory variables between control and pRP patients is demonstrated in Table 1.

Serum hemoglobin, platelet, creatinine, ALT, D-dimer, FAR, NLR, albumin, and DDAR values were found to be similar between the groups $(p>0.05)$. Slightly higher AST values $(p=0.027)$ were detected in the pRP group $(25.60 \pm 9.77 \mathrm{IU} / \mathrm{L})$, when compared with the healthy subjects $(21.20 \pm 5.14 \mathrm{IU} / \mathrm{L})$. Serum WBC, MPV, fibrinogen, and MCP1 values were significantly higher in the pRP group (Table 1), when compared with the healthy control group $(p<0.05)$.
Higher MCP-1 values (Figure 2) were detected in participants with an abnormal FMD response $(p=0.001)$. A significant correlation was detected between abnormal FMD responses and MCP-1 levels (R: 0.308, $\mathrm{R}^{2}: 0.095, p$ : 0.044). In addition, a low-level relationship was found between FMD response and MPV, MCP-1, and fibrinogen values (Table 2).

We studied abnormal FMD, WBC, and MCP parameters by using the binary logistic regression model. Nagelkerke's $R^{2}$ determination coefficient was used to evaluate the predictive ability of the model. This model correctly predicted a control value of $83.3 \%$ and Raynaud's phenomenon of $86.0 \%$ (Table 3 ). The Nagelkerke $R^{2}$ value explains $64.3 \%$ of the variance to distinguish whether it is Raynaud's or not.

When the WBC and MCP-1 values were analyzed using the ROC curve method, the optimum diagnostic cutoff point was found to be $6.8210^{-3} / \mathrm{uL}$ for WBC with a sensitivity of $67.4 \%$ and specificity of $80 \%$ and the optimum diagnostic cutoff point was found for MCP-1 at $273.30 \mathrm{pg} / \mathrm{ml}$, with a sensitivity of $97.7 \%$ and a specificity of $60 \%$ (Figure 3 ).

\section{Discussion}

To the best of our knowledge, this is the first study examining the relationship between FMD response (impaired endothelial response) and blood parameters in patients with pRP. This is also the first report regarding the relationship between impaired endothelial responses and MCP-1 levels in pRP patients. Our results have indicated that patients with $\mathrm{pRP}$ have higher impaired endothelial response to ischemia. Incremental levels of serum WBC, MPV, MCP-1, and fibrinogen values were detected in the pRP group. Moreover, a moderate direct correlation was found between abnormal FMD responses and serum MCP-1 values. In multiple binary logistic regression analyses, WBC and MCP-1 were determined to be the most meaningful blood parameters for identifying pRP patients, with acceptable sensitivity and specificity rates.

Some of the predicted parameters in the distinction of pRP and sRP are age and gender. The first symptom attacks usually begin under the age of 30 for $\mathrm{pRP}$ and are generally observed more frequently in the female gender. In our study, the mean age of the patient group was found to be $28.33 \pm 8.71$, which is consistent with the literature, and the female gender was predominant (79\%) [16]. The impaired endothelial dysfunction has been demonstrated in both primary and secondary RP in previous studies. It has also been shown that microvascular derangement was associated with endothelial dysfunction in RP patients $[17,18]$. The endothelium has an important role in the regulation of vascular response against blood flow-associated shear stress and ischemia-reperfusion events. Insufficient flow-mediated vasorelaxation response is an indicator for increased vascular disease [19]. The dilatation response of the brachial artery after a 4-to-5 minute ischemic period is a reliable, noninvasive assessment method for the evaluation of endothelium functions. The postischemic brachial artery dilatation equal to or greater than $5 \%$ of normal diameter is accepted as a normal endothelial function, whereas dilatation with a lower than $5 \%$ diameter is accepted as impaired endothelial functions $[13,20]$. 


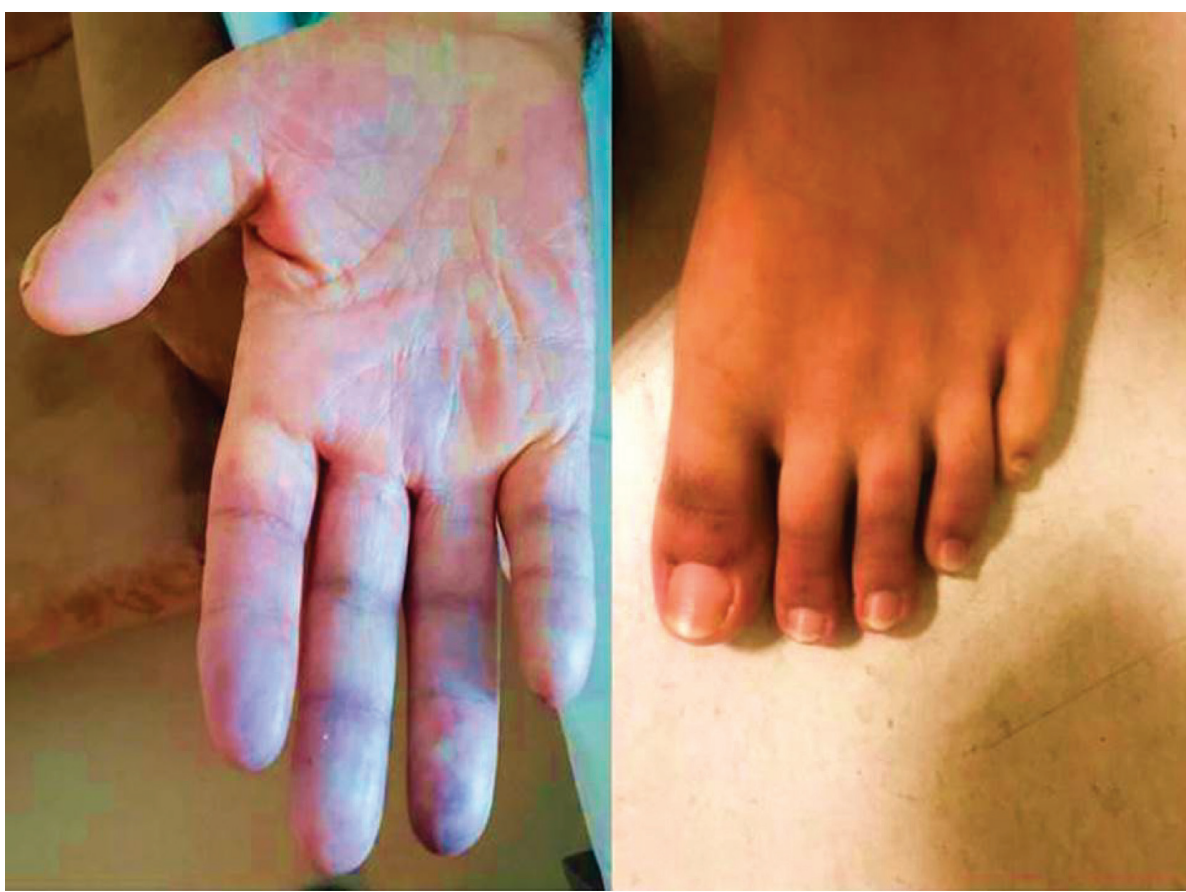

FIGURE 1: Bruising on the hand and foot in patients with pRP.

TABLE 1: Comparison of demographic and laboratory variables in pRP and healthy control groups.

\begin{tabular}{lccc}
\hline Variables & Control $(N: 30)$ & Raynaud's phenomenon $(N: 43)$ & $p^{\#}$ \\
\hline Female, $n(\%)$ & $22(73 \%)$ & $34(79 \%)$ & 0.772 \\
Age $($ mean \pm SD) & $27.17 \pm 8.01$ & $28.33 \pm 8.71$ & $19(44 \%)$ \\
Smoking, $n(\%)$ & $8(27 \%)$ & $30(70 \%)$ & 0.565 \\
Abnormal FMD, $n(\%)$ & $5(17 \%)$ & $7.64 \pm 1.73$ & 0.201 \\
WBC $\left(10^{-3} / \mu \mathrm{L}\right)$ & $6.20 \pm 1.43$ & $12.93 \pm 1.78$ & $\mathbf{0 . 0 0 1}$ \\
Hemoglobin $(\mathrm{g} / \mathrm{dL})$ & $13.46 \pm 1.06$ & $250.91 \pm 74.67$ & 0.148 \\
Platelet count $\left(10^{-3} / \mu \mathrm{L}\right)$ & $225.50 \pm 79.23$ & $10.47 \pm 0.87$ & 0.167 \\
MPV $(\mathrm{fL})$ & $8.36 \pm 0.96$ & $25.60 \pm 9.77$ & $\mathbf{0 . 0 0 1}$ \\
Creatinine $(\mathrm{mg} / \mathrm{dL})$ & $0.85 \pm 0.17$ & $26.12 \pm 12.94$ & 0.232 \\
AST $(\mathrm{IU} / \mathrm{L})$ & $21.20 \pm 5.14$ & $2.50 \pm 5.049$ & $\mathbf{0 . 0 2 7}$ \\
ALT $(\mathrm{IU} / \mathrm{L})$ & $23.45 \pm 7.36$ & $375.57 \pm 160.49$ & 0.311 \\
D-dimer $(\%)$ & $1.25 \pm 1.15$ & $112.54 \pm 45.49$ & $8.07 \pm 6.03$ \\
Fibrinogen $(\mu \mathrm{g} / \mathrm{ml})$ & $258.94 \pm 89.74$ & $3.3279 \pm 0.70561$ & $\mathbf{0 . 0 0 1}$ \\
FAR $(\%)$ & $88.38 \pm 66.85$ & $0.9210 \pm 1.65428$ & 0.090 \\
NLR $(\%)$ & $9.44 \pm 11.26$ & $332.4933 \pm 39.45015$ \\
Albumin $(\mathrm{g} / \mathrm{dL})$ & $3.3500 \pm 0.38841$ & 0.501 \\
DDAR $(\%)$ & $0.3819 \pm 0.37301$ & 0.579 \\
MCP-1 $(\mathrm{pg} / \mathrm{ml})$ & $264.4342 \pm 71.12647$ & 0.877 \\
\hline
\end{tabular}

FMD: flow-mediated dilatation; WBC: white blood cell; MPV: main platelet volume; AST: aspartate aminotransferase; ALT: alanine aminotransferase; FAR: fibrinogen-to-albumin ratio; NLR: neutrophil-to-lymphocyte ratio; DDAR: D-dimer-to-albumin ratio; MCP-1: monocyte chemoattractant protein-1. ${ }^{\#} p<0.05$ is considered as statistically significant. The bold numbers show the $p$ values below 0.05 .

The FMD response has been studied in patients with RP, albeit on small populations. Flavahan has indicated that an FMD response is normal in pRP and added that cold-induced disruption of arteriovenous connections in RP cases can lead to digital artery spasm [21]. Mavrikakis et al. studied FMD in patients with sRP, and they detected an abnormal FMD response in these patients. In addition, they found that ascorbic acid does not reverse endothelial vasomotor dysfunction in their study [22]. Klein-Weigel et al. investigated the seasonal variations in patients with RP, and they did not show an abnormal FMD response in pRP [23]. Although our results indicated that an abnormal FMD response was detected in $5(17 \%)$ of the 30 healthy individuals, the abnormal FMD was detected in $30(70 \%)$ of the 43 pRP patients.

Routine blood parameters have been investigated for several kinds of vascular diseases [24]. Platelet indices have been investigated in vasospastic disorders detected with a cold stimulation test by Kadan et al. [25]. They found a relationship between the severity of disease and MPV. Shemirani et al. found an independent relationship between pRP and higher serum MPV levels [26]. Another study on higher MPV suggested it was a triggering factor for $\mathrm{pRP}$ [27]. 


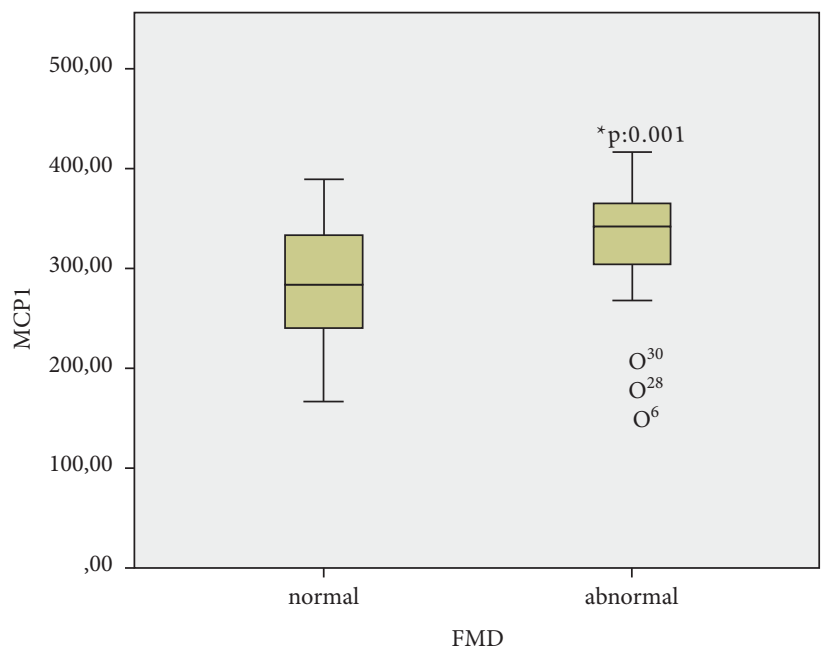

Figure 2: Comparison of monocyte chemoattractant protein-1 (MCP-1) values in normal and abnormal flow-mediated dilatation (FMD) groups.

TABLE 2: Relation between laboratory parameters and abnormal flow-mediated dilatation (FMD).

\begin{tabular}{|c|c|c|}
\hline & FMD & $p$ \\
\hline \multirow{4}{*}{ MCP-1 } & $R$ & 0.308 \\
\hline & $R^{2}$ & 0.095 \\
\hline & $p$ & 0.044 \\
\hline & $N$ & 43 \\
\hline \multirow{4}{*}{ WBC } & $R$ & 0.036 \\
\hline & $R^{2}$ & 0.001 \\
\hline & $p$ & 0.819 \\
\hline & $N$ & 43 \\
\hline \multirow{4}{*}{ MPV } & $R$ & 0.171 \\
\hline & $R^{2}$ & 0.029 \\
\hline & $p$ & 0.272 \\
\hline & $N$ & 43 \\
\hline \multirow{4}{*}{ Fibrinogen } & $R$ & 0.101 \\
\hline & $R^{2}$ & 0.010 \\
\hline & $p$ & 0.517 \\
\hline & $N$ & 43 \\
\hline
\end{tabular}

FMD: flow-mediated dilatation; MCP-1: monocyte chemoattractant protein-1; WBC: white blood cell; MPV: mean platelet volume; $p$ : correlation is significant at the 0.05 level (2-tailed). The bold numbers show the $p$ values below 0.05 .

In the same study, WBC levels were found to be an insignificant predictor. Lau et al. found increased white blood cell activation in patients with RP [28]. Our own findings supported that MPV and WBC levels are found in patients with pRP. Plasma fibrinogen is another laboratory parameter that was investigated in vasospastic disorders. Splenger et al. concluded that increased plasma fibrinogen levels in patients with RP are related to disrupted distal microcirculation [29]. Similarly, higher fibrinogen levels were found by Żuk et al. to be associated with plasma fibrin clots, displaying impaired lysability and increased endothelial damage in pRP [30]. In our study, higher fibrinogen levels were detected in $\mathrm{pRP}$ patients.

MCP-1 is an important potent regulatory chemokine that is responsible for the migration and the infiltration of monocytes. Ischemia, oxidative stress, released cytokines, or growth factors trigger the biological activity of MCP-1 and lead to infiltration of monocytes/macrophages [31]. Rajagopalan et al. investigated MCP-1 levels in SRP and pRP, and they found that MCP-1 levels were higher in sRP, when compared with the primary type. However, they did not compare the MCP-1 levels with healthy controls and did not investigate the relation with endothelial response [31]. MCP1 was suspected in the pathogenesis of vasospastic outcomes of diseases that presented with sRP $[32,33]$. However, the literature includes insufficient reports about the relationship between impaired endothelial function and MCP-1 levels in pRP. Furthermore, another insufficiency exists regarding the differences between pRP and normal populations, in regard to MCP-1 levels. Our results indicate that MCP-1 levels are higher in patients with $\mathrm{pRP}$, and these results reveal a relationship between impaired endothelial functions and serum MCP-1 levels.

In conclusion, our findings demonstrated that MPV, WBC, fibrinogen, and MCP-1 levels were higher in pRP patients, when compared with the healthy control. It appears that increased MCP-1 values were related to abnormal FMD responses in the pRP group. These results might be helpful for the establishment of the pathophysiology of the RP.

4.1. Limitations of the Study. The main limitation of the study is concerning the small sample size. Although previous studies have presented findings with smaller patient groups, larger comprehensive cohorts are required to obtain compelling and precise results. The second limitation is concerning the determination of endothelial response with a single method (FMD). The endothelial response should be confirmed with other techniques in order to present clear findings. The third limitation is related to the nature of the disease: if no underlying disease is determined to be responsible for RP, then it is called primary RP. However, there is a multifactorial etiology for RP, and it is therefore possible to omit a proper diagnosis. 
TABLE 3: Multiple binary logistic regression analysis.

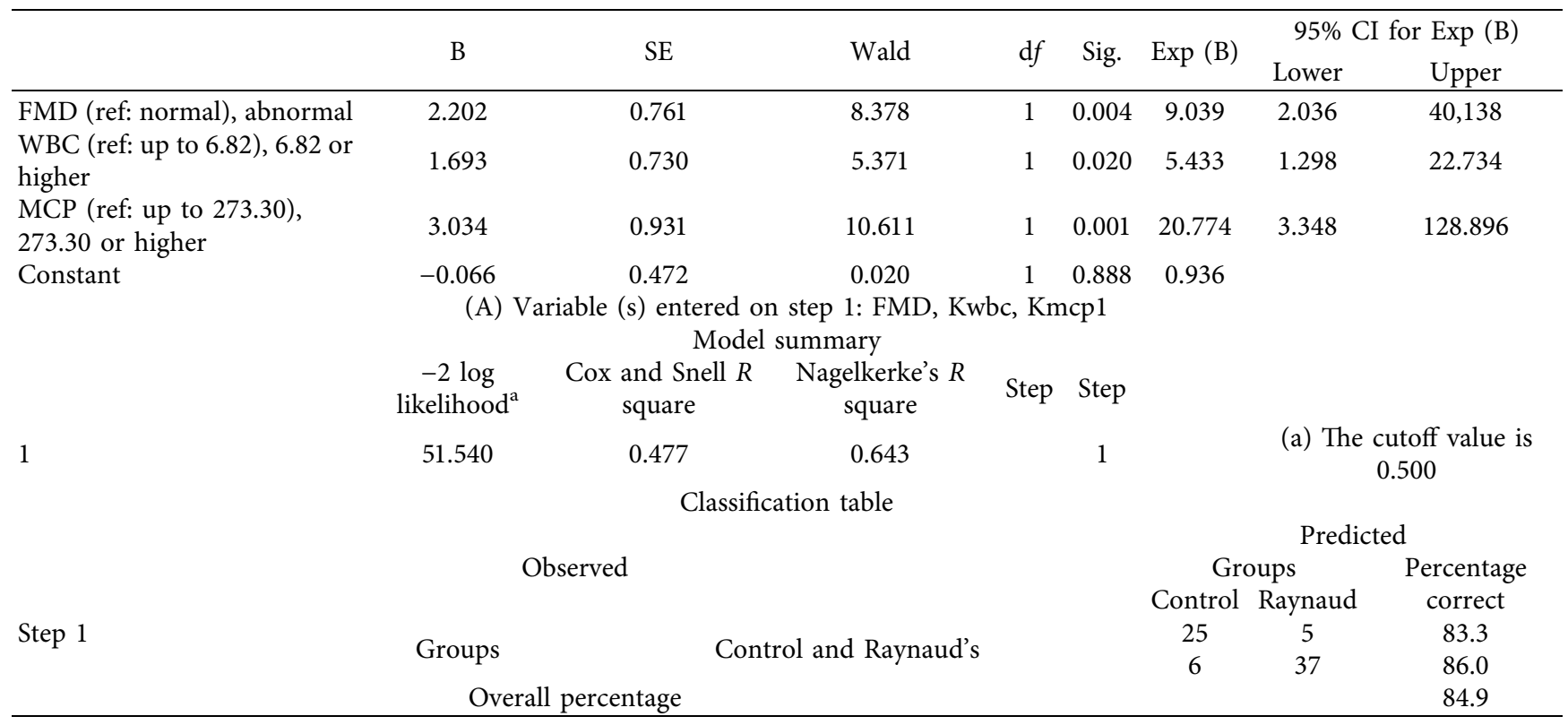

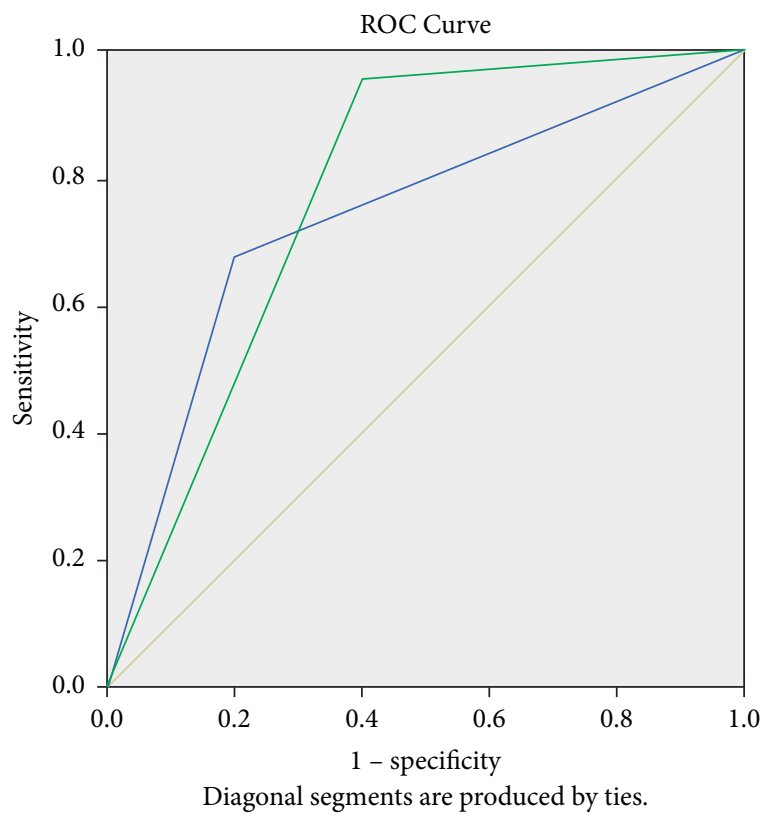

Source of the Curve

— KatWbc

— KatMcp1

Reference Line

\begin{tabular}{|c|c|c|c|c|c|}
\hline RISK FACTOR & AUC (\%95) & cut off & p & sensitivity (\%) & specificity (\%) \\
\hline WBC & $0.743(0.626-0.853)$ & 6.82 & 0.000 & $67.4 \%$ & $80,00 \%$ \\
\hline MCP1 & $0.781(0.660-0.901)$ & 273.30 & 0.000 & $97.7 \%$ & $60,00 \%$ \\
\hline
\end{tabular}

FIGURE 3: ROC analysis of the serum WBC and MCP-1 values for prediction of primary Raynaud's phenomenon. 


\section{Data Availability}

The data used to support the findings of this study are included within the article.

\section{Disclosure}

This manuscript has been released as a preprint at https:// www.authorea.com/users/429233/articles/532964-theevaluation-of-flow-mediated-dilatation-and-bloodparameters-in-raynaud-phenomenon [34].

\section{Conflicts of Interest}

The authors declare that they have no conflicts of interest.

\section{Authors' Contributions}

SU had substantial contributions to the conception or design of the work; the acquisition, analysis, or interpretation of data for the work; drafting of the work or critical revision of the manuscript for important intellectual content; and the final approval of the version to be published. IK was responsible for drafting the work or revising it critically for important intellectual content; agreement to be accountable for all aspects of the work in ensuring that questions related to the accuracy or integrity of any part of the work are appropriately investigated and resolved; and the final approval of the version to be published.

\section{References}

[1] F. Ingegnoli, N. Ughi, C. Crotti, M. Mosca, and C. Tani, "Outcomes, rates and predictors of transition of isolated Raynaud's phenomenon: a systematic review and metaanalysis," Swiss Medical Weekly, vol. 147, no. 3940, p. w14506, 2017.

[2] M. Hughes, Y. Allanore, L. Chung, J. D. Pauling, C. P. Denton, and M. Matucci-Cerinic, "Raynaud phenomenon and digital ulcers in systemic sclerosis," Nature Reviews Rheumatology, vol. 16, no. 4, pp. 208-221, 2020.

[3] F. Galluccio and M. Matucci-Cerinic, "Two faces of the same coin: Raynaud phenomenon and digital ulcers in systemic sclerosis," Autoimmunity Reviews, vol. 10, no. 5, pp. 241-243, 2011.

[4] D. Carlsson, J. Wahlström, L. Burström et al., "Can sensation of cold hands predict Raynaud's phenomenon or paraesthesia?" Occupational Medicine, vol. 68, no. 5, pp. 314-319, 2018.

[5] K. K. Temprano, “A review of Raynaud's disease," Missouri Medicine, vol. 113, no. 2, pp. 123-126, 2016.

[6] S. N. Lambova and U. Müller-Ladner, "The role of capillaroscopy in differentiation of primary and secondary Raynaud's phenomenon in rheumatic diseases: a review of the literature and two case reports," Rheumatology International, vol. 29, no. 11, pp. 1263-1271, 2009.

[7] J. Latuskiewicz-Potemska, A. Chmura-Skirlinska, R. J. Gurbiel, and E. Smolewska, "Nailfold capillaroscopy assessment of microcirculation abnormalities and endothelial dysfunction in children with primary or secondary Raynaud syndrome," Clinical Rheumatology, vol. 35, no. 8, pp. 1993-2001, 2016.
[8] A. Tuncay and D. Elcik, "The effect of neutrophil/lymphocyte ratio and tomography measurements at the time of diagnosis in predicting aneurysm rupture in patients with abdominal aortic aneurysms," Turkish Journal of Vascular Surgery, vol. 30, no. 1, pp. 1-6, 2021.

[9] N. Kankilic, A. Aslan, O. Karahan, S. Demirtas, A. Caliskan, and C. Yavuz, "Investigation of the arterial intima-media thickness in Behcet's disease patients without vascular complaints," Vascular, vol. 26, no. 4, pp. 356-361, 2018.

[10] C. Yavuz, A. Caliskan, O. Karahan, S. Demirtas, and S. Yazici, "Diagnostic accuracy of mean platelet volume in thromboangiitis obliterans," Vascular, vol. 22, no. 1, pp. 42-45, 2014.

[11] A. Duman, K. A. Turkdogan, M. Avcil et al., "The predictive value of the inflammatory markers P-selectin and MCP1 in determining the length of stay and 30-day survival in the differentiation of sepsis patients," JPMA. The Journal of the Pakistan Medical Association, vol. 68, no. 9, pp. 1321-1326, 2018.

[12] M. C. Corretti, T. J. Anderson, E. J. Benjamin et al., "Guidelines for the ultrasound assessment of endothelialdependent flow-mediated vasodilation of the brachial artery," Journal of the American College of Cardiology, vol. 39, no. 2, pp. 257-265, 2002.

[13] O. Karahan, S. Manduz, G. Bektasoglu, A. Zorlu, K. A. Turkdogan, and S. Bozok, "A high oxidative stress index predicts endothelial dysfunction in young male smokers," Bratislava Medical Journal, vol. 114, no. 12, pp. 721-725, 2013.

[14] C.-S. Kim, H.-S. Park, T. Kawada et al., "Circulating levels of MCP-1 and IL- 8 are elevated in human obese subjects and associated with obesity-related parameters," International Journal of Obesity, vol. 30, no. 9, pp. 1347-1355, 2006.

[15] S. Avnioglu, S. Avnioglu, N. Kozac1, and H. Yılmaz, "Comparison of total heart volume and laboratory results in COVID-19 infection," Biomedical Journal of Scientific \& Technical Research, vol. 38, no. 3, 2021 pages, 2021.

[16] B. Goundry, L. Bell, M. Langtree, and A. Moorthy, "Diagnosis and management of Raynaud's phenomenon," BMJ, vol. 344, p. e289, 2012.

[17] J. H. Le and K. I. Cho, “Association between endothelial function and microvascular changes in patients with secondary Raynaud's phenomenon," Clinical Rheumatology, vol. 33, no. 11, pp. 1627-1633, 2014.

[18] B. Mosdósi, K. Bölcskei, and Z. Helyes, "Impairment of microcirculation and vascular responsiveness in adolescents with primary Raynaud phenomenon," Pediatric Rheumatology, vol. 16, no. 1, p. 20, 2018.

[19] K. E. Pyke and M. E. Tschakovsky, "The relationship between shear stress and flow-mediated dilatation: implications for the assessment of endothelial function," The Journal of Physiology, vol. 568, no. 2, pp. 357-369, 2005.

[20] M. Charakida, S. Masi, T. F. Luscher, J. J. P. Kastelein, and J. E. Deanfield, "Assessment of atherosclerosis: the role of flow-mediated dilatation," European Heart Journal, vol. 31, no. 23, pp. 2854-2861, 2010.

[21] N. A. Flavahan, "A vascular mechanistic approach to understanding Raynaud phenomenon," Nature Reviews Rheumatology, vol. 11, no. 3, pp. 146-158, 2015.

[22] M. E. Mavrikakis, J. P. Lekakis, M. Papamichael, K. S. Stamatelopoulos, C. C. Kostopoulos, and S. F. Stamatelopoulos, "Ascorbic acid does not improve endothelium-dependent flow-mediated dilatation of the brachial artery in patients with Raynaud's phenomenon secondary to systemic sclerosis," International Journal for Vitamin and Nutrition Research, vol. 73, no. 1, pp. 3-7, 2003. 
[23] P. Klein-Weigel, K. Krall, J. Falkensammer, P. Heinz-Erian, H. Ulmer, and G. Fraedrich, "Die flowmediierte Dilatation der Brachialarterie von Frauen mit primären Raynaud Phänomen und gesunden Probanden ist nicht jahreszeitabhängig," Vasa, vol. 32, no. 2, pp. 69-73, 2003.

[24] O. Karahan, C. Yavuz, N. Kankilic et al., "Simple blood tests as predictive markers of disease severity and clinical condition in patients with venous insufficiency," Blood Coagulation and Fibrinolysis, vol. 27, no. 6, pp. 684-690, 2016.

[25] U. Demirkılıç, K. Karabacak, E. Kaya, G. Arslan, G. Erol, and S. Doğanc1, "Platelet indices may be correlated with severity of vasospastic disorders," Medical Science Monitor Basic Research, vol. 21, pp. 63-67, 2015.

[26] A.-H. Shemirani, B. Nagy Jr., A.-T. Takáts et al., "Increased mean platelet volume in primary Raynaud's phenomenon," Platelets, vol. 23, no. 4, pp. 312-316, 2012.

[27] E. Turan and S. S. Kilic, "Retrospective view of primary Raynaud's phenomenon in childhood," Reumatología Clínica, vol. 15, no. 6, pp. e92-e95, 2019.

[28] C. S. Lau, A. O’Dowd, and J. J. Belch, "White blood cell activation in Raynaud's phenomenon of systemic sclerosis and vibration induced white finger syndrome," Annals of the Rheumatic Diseases, vol. 51, no. 2, pp. 249-252, 1992.

[29] M. I. Spengler, M. J. Svetaz, M. B. Leroux, M. L. Leiva, and H. M. Bottai, "Association between capillaroscopy, haemorheological variables and plasma proteins in patients bearing Raynaud's phenomenon," Clinical Hemorheology and Microcirculation, vol. 30, no. 1, pp. 17-24, 2004.

[30] J. Żuk, A. Snarska-Drygalska, K. P. Malinowski, E. PapugaSzela, J. Natorska, and A. Undas, "Unfavourably altered plasma clot properties in patients with primary Raynaud's phenomenon: association with venous thromboembolism," Journal of Thrombosis and Thrombolysis, vol. 47, no. 2, pp. 248-254, 2019.

[31] S. L. Deshmane, S. Kremlev, S. Amini, and B. E. Sawaya, "Monocyte chemoattractant protein-1 (MCP-1): an overview," Journal of Interferon and Cytokine Research, vol. 29, no. 6, pp. 313-326, 2009.

[32] S. Rajagopalan, D. Pfenninger, C. Kehrer et al., "Increased asymmetric dimethylarginine and endothelin 1 levels in secondary Raynaud's phenomenon: implications for vascular dysfunction and progression of disease," Arthritis \& Rheumatism, vol. 48, no. 7, pp. 1992-2000, 2003.

[33] T. Yamamoto and K. Nishioka, "Role of monocyte chemoattractant protein-1 and its receptor, CCR-2, in the pathogenesis of bleomycin-induced scleroderma," Journal of Investigative Dermatology, vol. 121, no. 3, pp. 510-516, 2003.

[34] S. Uzun and I. Kaya, "The evaluation of flow mediated dilatation and blood parameters in Raynaud phenomenon," 2020. Authorea [Preprint]. 